Tohoku J. Exp. Med., 2005, 207, 303-312

\title{
Does Handgrip Exercise Training Increase Forearm Ischemic Vasodilator Responses in Patients Receiving Hemodialysis?
}

\author{
Naomi Kuge, Tomio Suzuki ${ }^{1}$ and Shogen Isoyama \\ Faculty of Medical Science and Welfare, Tohoku Bunka Gakuen University, \\ Sendai, Japan and \\ ${ }^{I}$ Nursing Home Sensyuen, Sendai, Japan
}

\begin{abstract}
Kuge, N., Suzuki, T. and Isoyama, S. Does Handgrip Exercise Training Increase Forearm Ischemic Vasodilator Responses in Patients Receiving Hemodialysis? Tohoku J. Exp. Med., 2005, 207 (4), 303-312 — In patients receiving hemodialysis, exercise capacity is extremely limited. Although vasodilation is one of the key phenomena for blood perfusion into working skeletal muscles during exercise, it is not clear whether the vasodilator capacity is increased after physical training in this population. We attempted to clarify whether handgrip exercise training increases forearm vasodilator responses to arterial occlusion, and to determine the relationship between muscle contraction function and the vasodilator responses in patients receiving hemodialysis. Eight patients and 7 age-matched healthy controls were tested. The patients participated in handgrip training four times a week for 6 weeks. Before and after the training the maximum muscle strength and endurance were measured with a handgrip dynamometer, and the forearm vasodilator responses to 3-minute arterial occlusion were measured by the near infrared spectroscopy technique. Maximum strength and endurance were significantly lower in the patients than in the controls. Maximum strength (from $183 \pm 84$ to $228 \pm 92$ Newtons, $p<0.05$ ) and endurance (from $19 \pm 6$ to $31 \pm 8 \mathrm{sec}, p<0.05$ ) were both increased after the training in the patients. Vasodilator responses estimated by the ratio of the maximum value of oxyhemoglobin after relief of arterial occlusion to its minimum value before the relief were significantly smaller in the patients compared with those in the controls $(132 \pm 20$ vs $161 \pm 27 \%, p<$ $0.05)$. In contrast to the findings in muscle function, the decreased vasodilator responses were not improved after the training $(141 \pm 17 \%)$. Additionally, no improvement in the vasodilator responses was observed in the parameters estimated by oxygen saturation. These data suggest that exercise capacity increased by physical training is produced by the functional improvement of skeletal muscles per se, but not by alterations in blood perfusion for oxygenation of the muscles in patients receiving hemodialysis. — renal disease; physical training; skeletal muscle; reactive hyperemia near infrared spectroscopy (C) 2005 Tohoku University Medical Press
\end{abstract}

Received August 2, 2005; revision accepted for publication September 27, 2005.

Correspondence: Naomi Kuge, Ph.D., PT, Faculty of Medical Science and Welfare, Tohoku Bunka Gakuen University, 6-45-1 Kunimi, Aoba-ku, Sendai 980-8551, Japan.

e-mail: naomi@rehab.tbgu.ac.jp 
In patients receiving hemodialysis, exercise capacity is extremely limited, and poor physical functioning has been linked to low quality of life and high mortality in this population (Kouidi et al. 1998; Johansen 1999; Heiwe et al. 2001; DePaul et al. 2002). Abnormalities such as loss of skeletal muscle mass (Kouidi et al. 1998; Johansen 1999), severe degenerative changes in muscle fibers and mitochondria (Kouidi et al. 1998; Johansen 1999), and impaired energy metabolism (Thompson et al. 1996; Johansen 1999; Bellinghieri et al. 2003) have been observed by several investigators. In addition to the abnormalities in skeletal muscle fibers, decreased number of capillaries in the muscular tissue (Kouidi et al. 1998; Johansen 1999), diminished vasodilator capacity in response to ischemia (Pannier et al. 2000; London et al. 2004), and impaired endothelium-dependent vasodilation (Joannides et al. 1997; Oflaz et al. 2003) were also observed in such patients. Thus, in patients receiving hemodialysis, abnormal findings were observed both in working skeletal muscles per se and in the vasculature for oxygenation of the working skeletal muscles.

On the other hand, it has been reported that physical training increases exercise capacity (Kouidi et al. 1998; Heiwe et al. 2001; Konstantinidou et al. 2002), and improves muscle contraction function (Heiwe et al. 2001; Rus et al. 2003) with restoration of the muscle atrophy (Kouidi et al. 1998) and an increase in the number of mitochondria in the muscle fibers (Kouidi et al. 1998). Furthermore, increases in the number of capillaries (Kouidi et al. 1998) and in the size of forearm large arteries and veins with functional improvement of the endothelium (Leaf et al. 2003; Rus et al. 2003) after physical training were reported. However, it is not clear whether the vasodilator capacity is increased after physical training, although vasodilation is one of the key phenomena for blood perfusion into working skeletal muscles during exercise.

In this study, therefore, we attempted to clarify whether handgrip exercise training increases the forearm vasodilator responses to arterial occlusion, and to determine the relationship between the muscle contraction function and the vasodilator responses after the training in patients receiving hemodialysis.

\section{Methods}

Subjects

Patients were eligible for inclusion when: (i) they had been on hemodialysis at least for 30 months $(93 \pm 65$ months, mean \pm S.D.); (ii) they had no clinically evident cardiovascular disease or medical problems that contraindicate participation in physical training; and (iii) they agreed to participate in this study which was approved by the ethics committee of Tohoku Bunka Gakuen University. In all, 8 patients (6 males and 2 females) fulfilled the above criteria. The underlying causes for renal failure were chronic nephritis in two patients, polycystic kidney disease in one patient, and diabetes mellitus in five patients. In addition, 7 healthy volunteers without renal disease ( 3 males and 4 females) were included for the age-matched control group after giving informed consent.

\section{Measurements of muscle strength and endurance}

Measurements were performed in a temperaturecontrolled room $\left(22-24^{\circ} \mathrm{C}\right)$ in the afternoon. The subject sat on a chair, and the maximum strength of forearm muscles was determined with a handgrip dynamometer (SPR 6800, Sakai Co., Tokyo). The measurement was made on the hand without arterio-venous shunt for hemodialysis in each subject. The subject was forced to continue to grip the dynamometer as strongly as possible. Muscle endurance was defined as the period from initiation of muscle contraction to the time when the strength declined to $60 \%$ of its maximum value (Fig. 1).

\section{Measurements by near infrared-spectroscopy technique}

Measurements by near infrared-spectroscopy (NIRS) is a noninvasive optical method for continuous measurement of tissue oxygenation and hemodynamics (Delpy et al. 1988; De Blasi et al. 1994). In this study, we estimated the changes in the forearm before, during and after 3-minute arterial occlusion. The technique is based on the following fundamental characteristics: (i) the relative transparency of human tissue to light in the near infrared region $(700-1,000 \mathrm{~nm})$ and (ii) the oxygenation-dependent absorption of oxy-hemoglobin and -myoglobin, and deoxy-hemoglobin and -myoglobin. Because of the overlap of the spectra, it is not possible to distinguish between changes in hemoglobin and myoglo- 


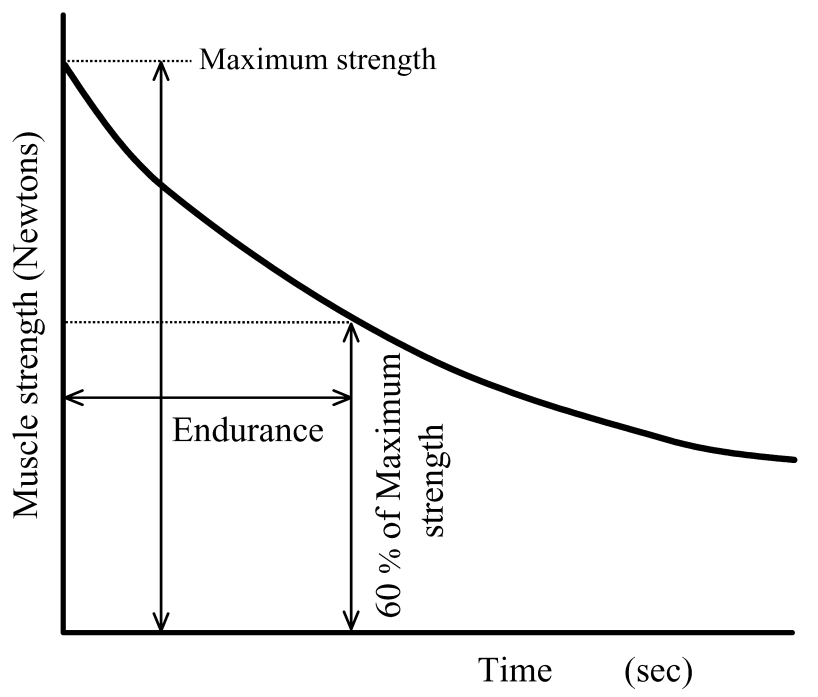

Fig. 1. Measurements of forearm muscle strength and endurance. The strength and endurance were measured using a handgrip dynamometer before and after 6-week handgrip exercise training. Subjects were forced to continue to grip it as strongly as possible (maximum muscle strength) and to maintain the maximum strength. Muscle endurance was defined as from the initiation of muscle contraction to the time when the strength declined to $60 \%$ of its maximum value.

bin. For convenience, both hemoglobin and myoglobin are referred to as hemoglobin in this study. By measuring changes in light absorption at different wavelengths $(780,810$ and $830 \mathrm{~nm})$ changes in tissue oxygenation can be measured continuously (Kawaguchi et al. 2001). The relation between light absorption and concentration changes of chromophores is described by the modified Beer-Lambert law (Delpy et al. 1988).

A BOM-L1TR instrument (Omega Wave Co., Tokyo) was used for simultaneous monitoring of concentration changes of oxy- and deoxyhemoglobin, and tissue blood oxygen saturation. The sampling rate was $1 \mathrm{~Hz}$. A summation of the changes in the concentration of oxyand deoxy-hemoglobin provides a measure of changes in the total tissue hemoglobin, which reflects changes in the tissue blood volume.

Each subject was placed in a supine position for 20 min prior to evaluation of the forearm vasodilator responses. The pneumatic cuff of a sphygmomanometer was placed around the upper arm for arterial occlusion. The emitting and receiving optodes were attached on the flexor digitorum superficial muscle $3 \mathrm{~cm}$ distally from the medial epicondyle. The interoptode distance was 20 $\mathrm{mm}$. After $5 \mathrm{~min}$ of baseline measurements, the cuff was inflated, and its pressure was maintained at $260 \mathrm{mmHg}$ for $3 \mathrm{~min}$. Then, the cuff was rapidly deflated. It took about 15 min to complete the measurement.

In control subjects, we measured blood pressure with a sphygmomanometer at the opposite arm before arterial occlusion, at 3 minutes of the occlusion, and one minute after its relief. The arterial occlusion did not produce any changes in blood pressure $(119 \pm 8 / 70 \pm 4,121$ $\pm 9 / 69 \pm 5$, and $117 \pm 9 / 69 \pm 6 \mathrm{mmHg}$, respectively)

As illustrated in Fig. 2, we analyzed the vasodilator responses to 3-min arterial occlusion in terms of two

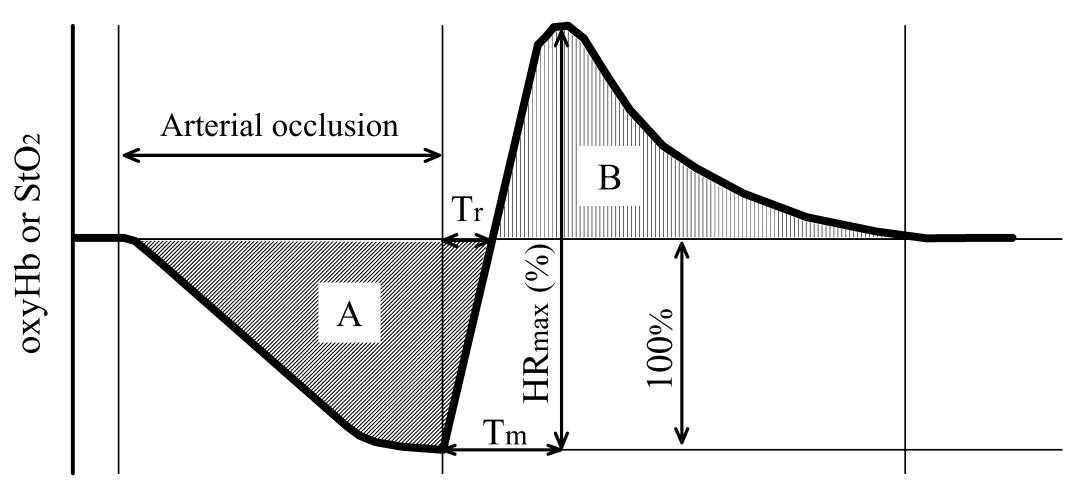

Fig. 2. Measurements of vasodilator responses to 3-min arterial occlusion by near-infrared spectroscopy technique. HRmax is defined as the ratio of the maximum value of oxyhemoglobin (oxyHb) or oxygen saturation $\left(\mathrm{StO}_{2}\right)$ after the relief of arterial occlusion to its minimum value during occlusion. HRarea is defined as the ratio of area A to area B. Tr and Tm are defined as the intervals between the relief of arterial occlusion and the recovery to baseline, and between the relief and the maximum value of the signal, respectively. 
magnitude parameters (HRmax and HRarea) and two time parameters ( $\mathrm{Tr}$ and Tmax). HRmax is defined as the ratio of the maximum value of oxyhemoglobin or oxygen saturation after the relief of arterial occlusion to its minimum value during occlusion. HRarea is defined as the ratio of area A to area $\mathrm{B}$. Tr and $\mathrm{Tm}$ are defined as the intervals between the relief of arterial occlusion and the recovery to baseline, and between the relief and the maximum value of the signal, respectively. Total hemoglobin and oxygen saturation were calculated as described below.

$$
\begin{aligned}
& \text { Total hemoglobin } \\
& \quad=\text { oxyhemoglobin + deoxyhemoglobin } \\
& \text { Oxygen saturation } \\
& \quad \text { = oxyhemoglobin/total hemoglobin }
\end{aligned}
$$

\section{Handgrip exercise training}

Each patient receiving hemodialysis underwent the handgrip exercise training for 6 weeks in our nursing home under the supervision of a physical therapist. The training was performed four times a week on the nondialysis days. In the first week, 50 repetitive handgrip contractions were applied at a strength of $60 \%$ of the maximum value which had been measured before starting the training. The repetitive contractions were increased up to 150 contractions by 20 contractions a week thereafter. It took 15 to $30 \mathrm{~min}$ to accomplish each set of the training.

\section{Statistical analysis}

Values are expressed as mean \pm S.D. MannWitney's U-test was used to compare mean values between control subjects and patients receiving hemodialysis. Wilcoxon test was used to determine the statistical significance between the values before and after handgrip training in the patients. When the $p$ value was less than 0.05 , the difference was determined to be statistically significant.

\section{RESULTS}

The physical and clinical characteristics of the age-matched control subjects and the patients receiving hemodialysis are summarized in Table 1. There was no difference in age or height between the two groups, but the body weight was greater in the control subjects than in the patients. The hematocrit and hemoglobin were lower, and the serum creatinine, blood urea nitrogen and serum $\mathrm{K}$ were higher in the patients immediately before receiving hemodialysis than in the control subjects. However, those parameters were not changed during the 6 weeks of this study.

Fig. 3 shows typical tracings of muscle strength obtained from a control subject and a patient receiving hemodialysis before and after handgrip training. The maximum voluntary contraction in the patient was significantly lower than

TABLE 1. Physical and clinical characteristics of control subjects and patients receiving hemodialysis

\begin{tabular}{lccc}
\hline \multirow{2}{*}{ Variables } & $\begin{array}{c}\text { Control subjects } \\
(n=7)\end{array}$ & Pefore training & After training \\
\cline { 3 - 4 } & & $61.1 \pm 5.8$ & - \\
\hline Age (years) & $58.7 \pm 5.8$ & $153.6 \pm 7.3$ & - \\
Height $(\mathrm{cm})$ & $160.9 \pm 10.1$ & $42.4 \pm 7.8^{*}$ & $42.5 \pm 8.0^{*}$ \\
Body weight $(\mathrm{kg})$ & $59.3 \pm 11.5$ & $3.9 \pm 0.3$ & $3.9 \pm 0.3$ \\
Serum albumin $(\mathrm{g} / 100 \mathrm{ml})$ & $3.7 \pm 0.2$ & $35 \pm 3.9^{*}$ & $34 \pm 3.6^{*}$ \\
Hematocrit $(\%)$ & $45 \pm 5.4$ & $11.3 \pm 1.2^{*}$ & $11.3 \pm 1.0^{*}$ \\
Hemoglobin $(\mathrm{g} / 100 \mathrm{ml})$ & $15.3 \pm 1.8$ & $9.8 \pm 2.1^{*}$ & $9.7 \pm 1.9^{*}$ \\
Serum creatinine $(\mathrm{mg} / 100 \mathrm{ml})$ & $0.6 \pm 0.1$ & $74 \pm 16.7^{*}$ & $64 \pm 16.1^{*}$ \\
Blood Urea Nitrogen $(\mathrm{mg} / 100 \mathrm{ml})$ & $15 \pm 3.6$ & $141 \pm 1.7$ & $139 \pm 2.0$ \\
Na (mEq/liter) & $139 \pm 1.9$ & $5.1 \pm 0.6^{*}$ & $4.8 \pm 0.4^{*}$ \\
K (mEq/liter) & $4.2 \pm 0.4$ & $104 \pm 1.9$ & $103 \pm 2.4$ \\
Cl (mEq/liter) & $101 \pm 2.6$ &
\end{tabular}

Values are mean \pm S.D. ${ }^{*} p<0.05$ between age-matched control subjects and patients receiving hemodialysis. 

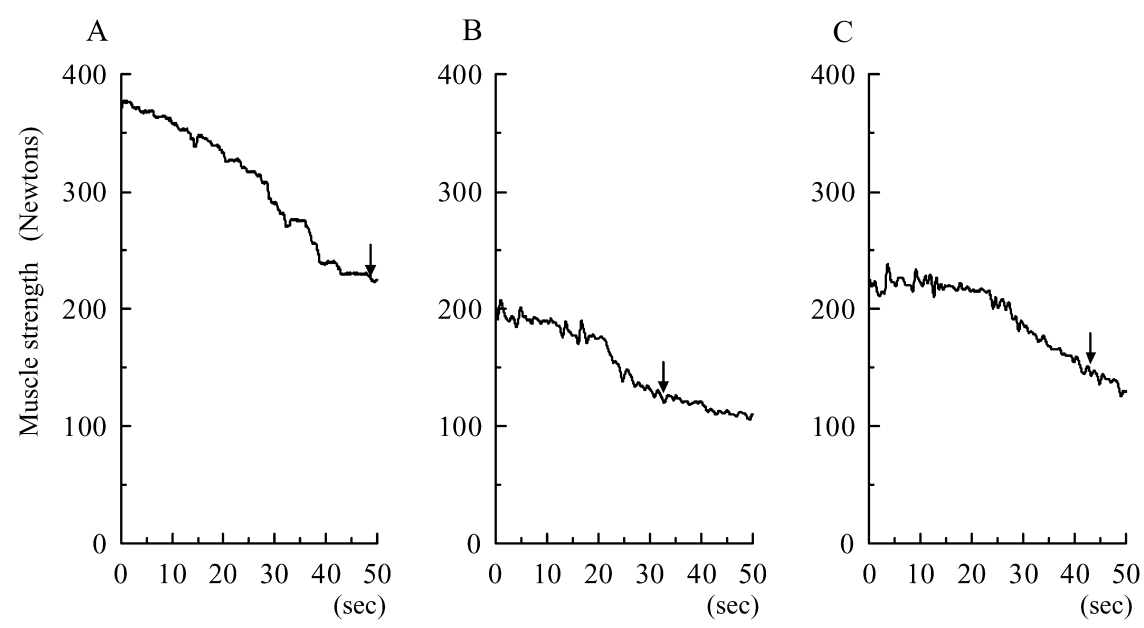

Fig. 3. Representative tracings of muscle strength in a control (Panel A) and a patient receiving hemodialysis before (Panel B) and after 6-week handgrip exercise training (Panel C). Arrows indicate the point of $60 \%$ of the maximum muscle strength which reflects muscle endurance.

that of the control subject (200 vs 378 Newtons). After the training, the value in the patient was increased to 226 Newtons. The shorter muscle endurance in the patient ( $32 \mathrm{vs} 48 \mathrm{sec}$ in the control subject) was also increased to $43 \mathrm{sec}$ after the training.

As shown in Fig. 4A, maximum muscle strength was significantly lower in the patients than in the control subjects $(183 \pm 84$ vs $366 \pm 82$ Newtons, $p<0.01)$, and was increased after the training ( $228 \pm 92$ Newtons, $p<0.05)$. As shown in Fig. 4B, muscle endurance was also significantly shorter in the patients than in the control subjects $(19 \pm 6$ vs $45 \pm 13 \mathrm{sec}, p<0.01)$, and was increased after the training $(31 \pm 8 \mathrm{sec}, p<$ 0.05).

Fig. 5 shows typical tracings of the vasodilator responses to 3-min arterial occlusion which were obtained from a control subject and a patient receiving hemodialysis before and after handgrip

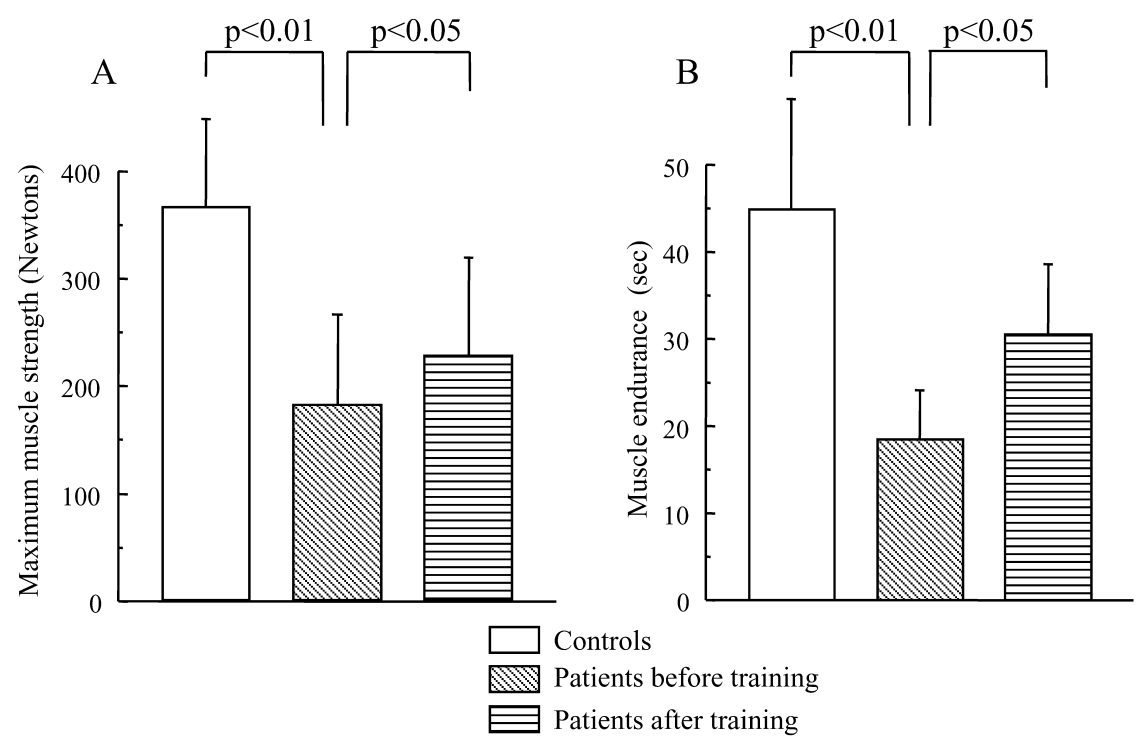

Fig. 4. Maximal muscle strength (Panel A) and endurance (Panel B) in the control subjects and patients before and after 6-week handgrip exercise training. Values are mean \pm S.D. 
A
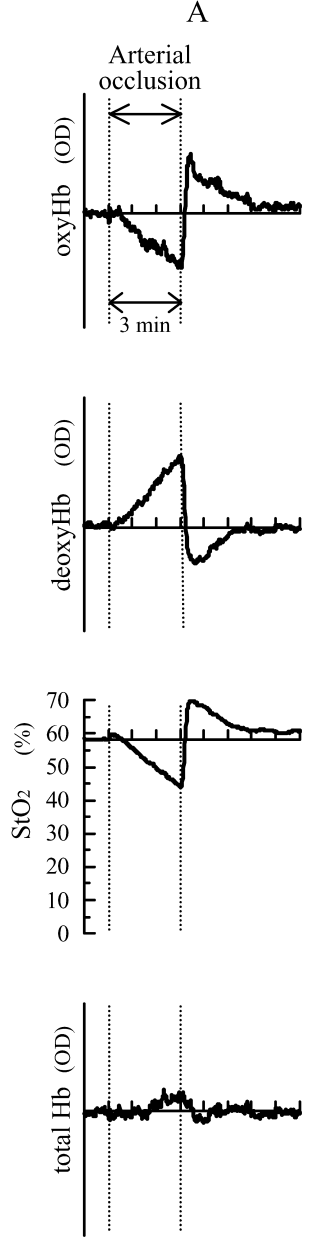

B
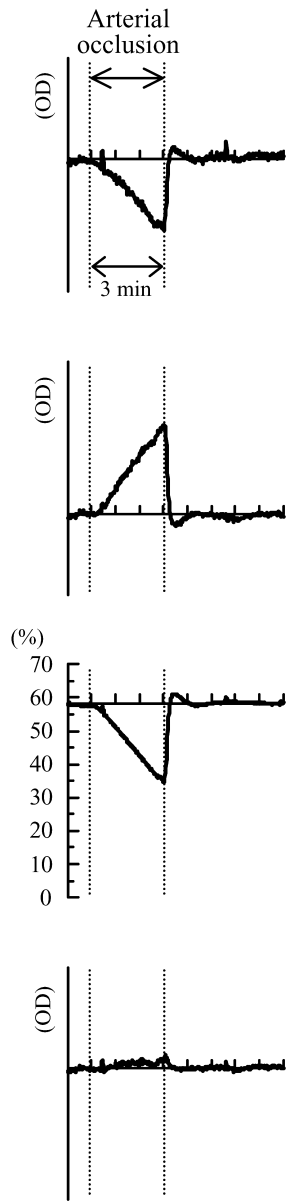
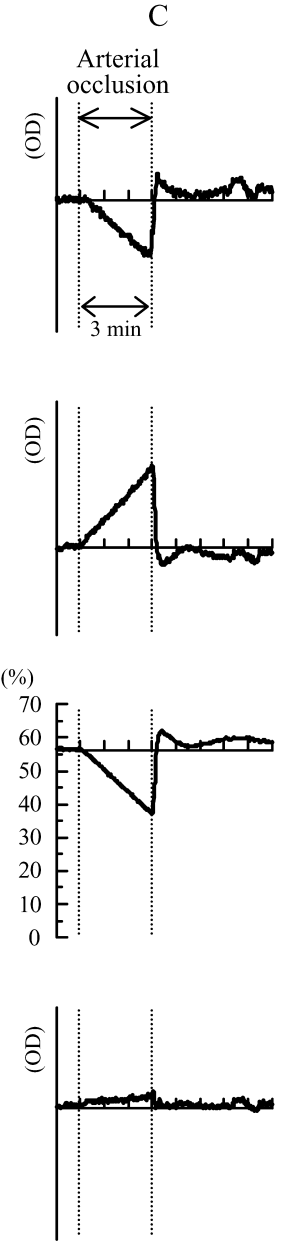

Fig. 5. Representative tracings of vasodilator responses to 3-min arterial occlusion using the near-infrared spectroscopy technique in a control (Panel A) and a patient before (Panel B) and after 6-week handgrip exercise training (Panel C). oxyHb, oxyhemoglobin; deoxyHb, deoxyhemoglobin; $\mathrm{StO}_{2}$, oxygen saturation.

exercise training. Forearm vasodilator responses (HRmax and HRarea) estimated from the changes in oxyhemoglobin and oxygen saturation were smaller in the patient receiving hemodialysis compared with those in the control subject. The vasodilator responses were not changed even after the training. There was no difference in the responses estimated by time parameters ( $\mathrm{Tr}$ or $\mathrm{Tm}$ ) between the control and the patient, or before and after the training in the patient. The tracings of deoxyhemoglobin showed mirror images of the tracings of oxyhemoglobin, and total hemoglobin did not significantly change throughout any measurement.

As shown in Fig. 6, HRmax estimated from the changes of oxyhemoglobin and oxygen saturation was significantly smaller in the patients than in the control subjects $(132 \pm 20$ vs $161 \pm$ $27 \%$ in oxyhemoglobin, $p<0.05 ; 131 \pm 14$ vs 156 $\pm 13 \%$ in oxygen saturation, $p<0.01$ ). Likewise, HRarea was smaller in the patients compared with that in the control subjects $(31 \pm 21$ vs $78 \pm 31 \%$ in oxyhemoglobin, $p<0.01 ; 28 \pm 17$ vs $54 \pm 16 \%$ in oxygen saturation, $p<0.05$ ). The diminished vasodilator responses were not altered even after the handgrip exercise training (HRmax: $141 \pm$ $17 \%$ in oxyhemoglobin and $135 \pm 13 \%$ in oxygen saturation; HRarea: $45 \pm 32 \%$ in oxyhemoglobin and $43 \pm 23 \%$ in oxygen saturation). There was no significant difference in responses estimated 

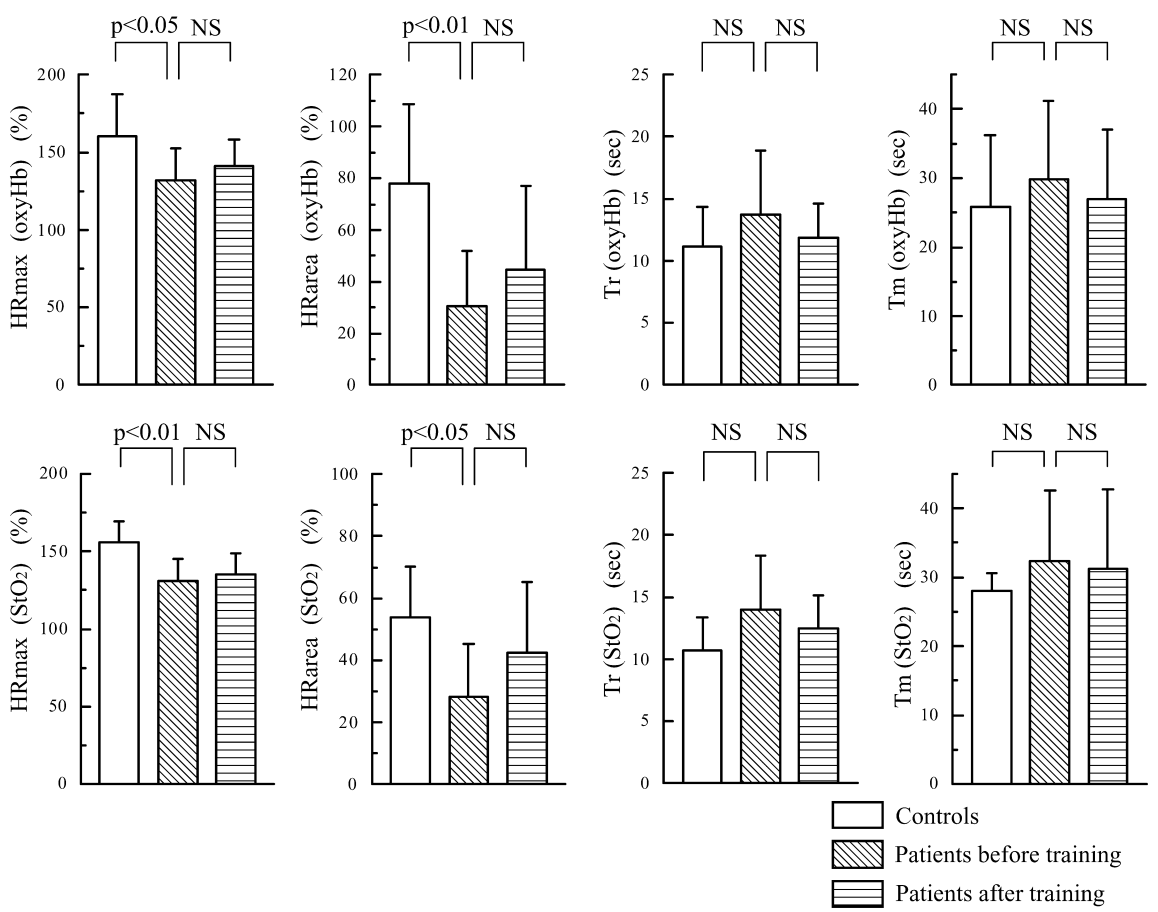

Fig. 6. Vasodilator responses to 3-min arterial occlusion in the control subjects and in the patients receiving hemodialysis before and after 6-week handgrip exercise training. Vasodilator responses were evaluated by the magnitude parameters, HRmax and HRarea, and the time parameters, $\operatorname{Tr}$ and $\mathrm{Tm}$, in the oxyhemoglobin (oxyHb) and oxygen saturation $\left(\mathrm{StO}_{2}\right)$ signals. Values are mean \pm S.D. NS, not significant.

by time parameters (Tmax or Tr) between the control subjects and the patients or between before and after the training in the patients.

\section{Discussion}

In the present study we examined the effects of 6-week handgrip exercise training on the forearm muscle contraction function and vasodilator responses to arterial occlusion in patients receiving hemodialysis.

Our purpose was to clarify the muscle function and vasodilator responses before and after the training in the patient group, but not to compare those changes between the control and patient groups after the training. Therefore, only the patient group underwent the training.

The muscle contraction and vascular dilation were both impaired in the patients compared with those in age-matched healthy controls. The training improved the maximum muscle strength and endurance in the patients, but it did not improve the decreased vasodilator responses to arterial occlusion, at least at 6 weeks after the initiation of training.

\section{Muscle function}

The contractile state alters the blood supply to the skeletal muscles through compressing the vascular trees by elevated intramuscular, extravascular pressure. In turn, the altered blood supply affects the muscle contraction function. Since we estimated the muscle contraction function by the parameters obtained during maximum contraction, the effect of blood perfusion on the muscle function would be minimal in the controls and patients, and before and after handgrip training in the patients.

In our patients receiving hemodialysis, muscle strength and endurance were both lower than those in age-matched healthy controls, and the strength and endurance were 50 and $42 \%$ of the respective values in the controls. This impaired 
muscle function has been observed and reported in previous studies (Thompson et al. 1996; Kouidi et al. 1998; Heiwe et al. 2001; DePaul et al. 2002). After handgrip exercise training, the strength was improved to $125 \%$ of the value before the training. The muscle endurance in the patients receiving hemodialysis was $42 \%$ of the value in the controls, and 6-week training increased the value to $163 \%$ of the value before the training. This beneficial effect of local physical training on the muscle function in the present study is consistent with the findings after local or systemic physical training in previous studies (Kouidi et al. 1998; DePaul et al. 2002; Headley et al. 2002; Konstantinidou et al. 2002; Oh-Park et al. 2002; Rus et al. 2003).

\section{Vascular function}

In the present study, using the NIRS technique we first estimated the vasodilator responses to arterial occlusion before and after physical training in patients receiving hemodialysis. This method rather than the venous (Pannier et al. 2000; London et al. 2004) or strain-guage (Tagawa et al. 1994) plethysmography technique for blood flow measurements enabled us to estimate the vascular responses of a quite limited area of locally trained muscles. The vascular responses to arterial occlusion estimated by the NIRS technique seem to be smaller than those estimated by blood flow measured by the plethysmography technique $(78 \%$ of HRarea estimated by oxyhemoglobin vs $116 \%$ of flow debt repayment after 5-minute arterial occlusion in control subjects (De Blasi et al. 1994; Pannier et al. 2000).

Regarding the functional and morphological vascular changes in patients receiving hemodialysis, several studies reported decreased vasodilator responses to arterial occlusion measured by plethysmography (decreased flow debt repayment [London et al. 2004] with shorter duration of hyperemia [Pannier et al. 2000]), diminished endothelium-dependent vasodilation endothelial dysfunction (Oflaz et al. 2003) and decreased NO availability in the endothelium (Joannides et al. 1997), and decreased microvascular density (Kouidi et al. 1998). After chronic physical train- ing, using ultrasound and duplex Doppler scanning Rus et al. (2003) and Leaf et al. (2003) reported that the size of forearm large arteries (Rus et al. 2003) and veins (Rus et al. 2003; Leaf et al. 2003) was significantly increased, and that the endothelium-dependent vasodilation was improved without changes in endotheliumindependent dilation (Rus et al. 2003). However, the effect of physical training on the vasodilator responses to arterial occlusion had not been clarified.

On the other hand, it has been reported that the endothelial function contributes to the basal arterial tone, to the postischemic hyperemic response in the human forearm (Tagawa et al. 1994; Meredith et al. 1996), and perhaps, to the forearm vasodilation during exercise (Loscalzo et al. 1994; Joyner and Dietz 1997). However, the contribution of the endothelial function to the hyperemic response was minimal, especially at the peak hyperemic response, but modest in maintaining vasodilation after hyperemia in healthy human forearms (Loscalzo and Vita 1994; Tagawa et al. 1994; Joyner et al. 1997). Hyperemic or vasodilator responses to ischemia are mainly determined by the microvascular function at the level of small arteries. In the present study, therefore, we studied the vasodilator responses to arterial occlusion using the NIRS technique before and after physical (handgrip) training in patients receiving hemodialysis.

We estimated the vasodilator responses to arterial occlusion by the signals of oxyhemoglobin and oxygen saturation. HRmax and HRarea were both decreased without changes in the time parameters ( $\mathrm{Tr}$ or $\mathrm{Tm}$ ) in patients receiving hemodialysis compared with those in the age-matched controls. The decreased vasodilator responses were not improved, at least 6 weeks after the initiation of handgrip exercise training. Pannier et al. (2000) and London et al. (2004) measured the flow debt repayment by venous plethysmography, and reported that the repayment in response to 5-min arterial occlusion was decreased to 87 $88 \%$ in patients receiving hemodialysis (vs $116 \%$ in the control subjects). Thus, the decreased vasodilator responses to arterial occlusion in our 
patients were consistent with their data.

In contrast to the beneficial effects of physical training on the endothelial function and arterial and venous size in the previous studies (Kouidi et al. 1998; Leaf et al. 2003; Rus et al. 2003) and on the muscle contraction function in the present study, 6-week handgrip training did not affect the forearm vasodilator responses. Several investigators reported the following effects of physical training on the vascular function. In middle-aged subjects, 8-week moderate intensity circuit training did not alter the endothelium-dependent or -independent vascular function while muscle strength was increased significantly (Maiorana et al. 2001). In patients receiving hemodialysis, 4 6 week handgrip exercise training had no effect on endothelium-dependent flow or peak reactive flow in response to 5-min arterial occlusion, but both were increased in control subjects (Bank et al. 1998). These data indicate that physical training does not always improve the vascular function including the vasodilator responses to arterial occlusion or ischemia, even if the muscle function is improved. In addition, the age or underlying disease of patients may be important factors in the improvement of vascular function after physical training. The type of physical training, i.e., isotonic or isometric, and local or systemic, and its severity and duration may also be important factors in improving the vascular function in patients (Headley et al. 2002; Konstantinidou et al. 2002).

\section{Interaction between muscle and vascular func- tion}

The muscle contraction function during exercise are determined by the characteristics of the muscles per se and indirectly by its oxygenation by the vasculature. Therefore, the vascular function including the vasodilator responses to ischemia is important for the patients receiving hemodialysis to increase the exercise capacity, which will result in better physical functioning and a higher quality of life for such patients. Our data suggest that exercise capacity increased by physical training is produced by the functional improvement of skeletal muscles per se, but not by alterations in blood perfusion for oxygenation of the muscles in patients receiving hemodialysis.

In summary, we first estimated vasodilator responses to ischemia before and after physical training in patients receiving hemodialysis. The training did not increase vasodilator capacity in spite of the improvement of muscle contraction function.

\section{References}

Bank, A.J., Shammas, R.A., Mullen, K. \& Chuang, P.P. (1998) Effects of short-term forearm exercise training on resistance vessel endothelial function in normal subjects and patients with heart failure. J. Card. Fail., 4, 193-201.

Bellinghieri, G., Santoro, D., Calvani, M., Mallamace, A. \& Savica, V. (2003) Carnitine and hemodialysis. Am. J. Kidney Dis., 41, Suppl. 1, S116-S122.

De Blasi, R.A., Ferrari, M., Natali, A., Conti, G., Mega, A. \& Gasparetto, A. (1994) Noninvasive measurement of forearm blood flow and oxygen consumption by near-infrared spectroscopy. J. Appl. Physiol., 76, 1388-1393.

Delpy, D.T., Cope, M., van der Zee, P., Arridge, S., Wray, S. \& Wyatt, J. (1988) Estimation of optical pathlength through tissue from direct time of flight measurement. Phys. Med. Biol., 33, 1433-1442.

DePaul, V., Moreland, J., Eager, T. \& Clase, C.M. (2002) The effectiveness of aerobic and muscle strength training in patients receiving hemodialysis and EPO: a randomized controlled trial. Am. J. Kidney Dis., 40, 1219-1229.

Headley, S., Germain, M., Mailloux, P., Mulhern, J., Ashworth, B., Burris, J., Brewer, B., Nindl, B., Coughlin, M., Welles, R. \& Jones, M. (2002) Resistance training improves strength and functional measures in patients with end-stage renal disease. Am. J. Kidney Dis., 40, 355-364.

Heiwe, S., Tollback, A. \& Clyne, N. (2001) Twelve weeks of exercise training increases muscle function and walking capacity in elderly predialysis patients and healthy subjects. Nephron, 88, 48-56.

Joannides, R., Bakkali, E.H., Le Roy, F., Rivault, O., Godin, M., Moore, N., Fillastre, J.P. \& Thuillez, C. (1997) Altered flow-dependent vasodilatation of conduit arteries in maintenance haemodialysis. Nephrol. Dial. Transplant., 12, 2623-2628.

Johansen, K.L. (1999) Physical functioning and exercise capacity in patients on dialysis. Adv. Ren. Replace Ther., 6, 141-148.

Joyner, M.J. \& Dietz, N.M. (1997) Nitric oxide and vasodilation in human limbs. J. Appl. Physiol., 83, 1785-1796.

Kawaguchi, K., Tabusadani, M., Sekikawa, K., Hayashi, Y. \& Onari, K. (2001) Do the kinetics of peripheral muscle oxygenation reflect systemic oxygen intake? Eur. J. Appl. Physiol., 84, 158-161.

Konstantinidou, E., Koukouvou, G., Kouidi, E., Deligiannis, A. \& Tourkantonis, A. (2002) Exercise training in patients with end-stage renal disease on hemodialysis: comparison of three rehabilitation programs. J. Rehabil. Med., 34, $40-45$.

Kouidi, E., Albani, M., Natsis, K., Megalopoulos, A., Gigis, P., Guiba-Tziampiri, O., Tourkantonis, A. \& Deligiannis, A. (1998) The effects of exercise training on muscle atrophy in haemodialysis patients. Nephrol. Dial. Transplant., 13, 685-699. 
Leaf, D.A., MacRae, H.S., Grant, E. \& Kraut, J. (2003) Isometric exercise increases the size of forearm veins in patients with chronic renal failure. Am. J. Med. Sci., 325, 115-119.

London, G.M., Pannier, B., Agharazii, M., Guerin, A.P., Verbeke, F.H. \& Marchais, S.J. (2004) Forearm reactive hyperemia and mortality in end-stage renal disease. Kidney Int., 65, 700-704.

Loscalzo, J. \& Vita, J.A. (1994) Ischemia, hyperemia, exercise, and nitric oxide. Complex physiology and complex molecular adaptations. Circulation, 90, 2556-2559.

Maiorana, A., O’Driscoll, G., Dembo, L., Goodman, C., Taylor, R. \& Green, D. (2001) Exercise training, vascular function, and functional capacity in middle-aged subjects. Med. Sci. Sports Exerc., 33, 2022-2028.

Meredith, I.T., Currie, K.E., Anderson, T.J., Roddy, M.A., Ganz, P. \& Creager, M.A. (1996) Postischemic vasodilation in human forearm is dependent on endothelium- derived nitric oxide. Am. J. Physiol., 270, H1435-H1440.

Oflaz, H., Pusuroglu, H., Genchallac, H., Demirel, S., Bugra, Z., Sever, M.S., \& Yildiz, A. (2003) Endothelial function is more impaired in hemodialysis patients than renal transplant recipients. Clin. Transplant., 17, 528-533.
Oh-Park, M., Fast, A., Gopal, S., Lynn, R., Frei, G., Drenth, R. \& Zohman, L. (2002) Exercise for the dialyzed: aerobic and strength training during hemodialysis. Am. J. Phys. Med. Rehabil., 81, 814-821.

Pannier, B., Guerin, A.P., Marchais, S.J., Metivier, F., Safar, M.E. \& London, G.M. (2000) Postischemic vasodilation, endothelial activation, and cardiovascular remodeling in endstage renal disease. Kidney Int., 57, 1091-1099.

Rus, R.R., Ponikvar, R., Kenda, R.B. \& Buturovic-Ponikvar, J. (2003) Effect of local physical training on the forearm arteries and veins in patients with end-stage renal disease. Blood Purif., 21, 389-394.

Tagawa, T., Imaizumi, T., Endo, T., Shiramoto, M., Harasawa, Y. \& Takeshita, A. (1994) Role of nitric oxide in reactive hyperemia in human forearm vessels. Circulation, 90, 2285-2290.

Thompson, R.T., Muirhead, N., Marsh, G.D., Gravelle, D., Potwarka, J.J. \& Driedger, A.A. (1996) Effect of anaemia correction on skeletal muscle metabolism in patients with end-stage renal disease: $31 \mathrm{P}$ magnetic resonance spectroscopy assessment. Nephron, 73, 436-441. 